\title{
The Lung-Gut-Cross Talk in Chronic Airways Diseases
}

\author{
Emiel F.M. Wouters, MD, PhD
}

Department Respiratory Diseases, Maastricht University Medical Center (MUMC) \& CIRO, Center of expertise for organ failure, Horn, The Netherlands

\section{ABSTRACT}

The gut and lung microbiota are essential to health and vital for the development of the immune system and homeostasis. The vital cross-talk between mucosal tissues of our body in chronic respiratory conditions as asthma, chronic obstructive pulmonary disease and cystic fibrosis in stable conditions as well as during exacerbations is still poorly studied and understood. The gut-lung interaction and the dysbiosis of microbiota and leaky guts in the aetiology of these chronic respiratory diseases are reviewed. (BRN Rev. 2019;5(1):19-34) Corresponding author: Emiel F.M. Wouters, e.wouters@mumc.nl

Key words: Asthma. Chronic obstructive pulmonary disease. Cystic fibrosis. Dysbiosis. Gut microbiome. Leaky gut. 


\section{INTRODUCTION}

The gut and respiratory tract epithelia have common anatomical similarities. Both are derived from the endoderm and consist of columnar epithelial cells with projection of microvilli or cilia that function as a physical barrier and as sentinels for the immune system in conjunction with associated lymphoid tissue. Otherwise, both epithelia have substantial differences. The intestinal lumen is an oxygen-poor environment and the movement of matter is unidirectional, from the mouth to the anus. The respiratory tract and alveoli are oxygen-rich and movement is bidirectional with no physical barrier between the larynx and the most distal alveolus. These different environments result in distinct microbial life in each organ ${ }^{1}$.

The intestinal tract represents not only a crucial component of the body's defence system against the external environment, but is also responsible for the breakdown and absorption of essential nutrients and the uptake of water and electrolytes. The barrier function of the intestinal tissue contributes to the first line of defence in the human body and is composed of a static epithelial and a dynamic luminal and subepithelial layer. Intestinal epithelial stem cells, residing at the base of crypt-villus axes, mature to various subtypes of intestinal epithelial cells. Besides absorptive enterocytes and mucus-secreting goblet cells, Paneth cells are specialised in maintaining the bacterial gradient along the crypt-villus axis by secretion of antimicrobial products. In addition, entero-endocrine cells connect the central nervous system with the neuro-endocrine system by secretion of hormones regulating digestive functions. This static epithelial barrier is composed by intestinal epithelial cells that interact with each other via a complex multilayer system. Tight junctions form a selectively permeable seal between adjacent epithelial cells. The dynamic part of the intestinal barrier is composed of the luminal microbiota and mucus as well as epithelial and immune cell products secreted into the lumen. The intestinal epithelium is furthermore covered by a thick mucus layer with large mucin glycoproteins as the major component, secreted by the goblet cells. Underneath the epithelial layer in the lamina propria, scattered intestinal immune cells and tissue specific organised lymphoid structures, such as the Peyer's patches are located. The immune response of the gut depends to a great extent on innate immune cells such as dendritic cells (DCs), monocytes/macrophages and the heterogeneous group of innate lymphoid cells in the intestine (Fig. 1).

There is growing clinical interest in secondary organ manifestations of chronic respiratory diseases and respiratory viral infections are often accompanied by intestinal symptoms ${ }^{2-6}$. Gastro-intestinal (GI) disease is more prevalent in patients with chronic obstructive pulmonary disease (COPD) than in healthy populations ${ }^{7,8}$. A population-based cohort showed a 2.72 times higher risk of Crohn's disease in COPD sufferers than that in healthy controls 9 . Vice versa is also reported: up to $50 \%$ of adults with inflammatory bowel disorders and one third of patients with irritable bowel syndrome have pulmonary involvement, such as inflammation or impaired lung function ${ }^{10,11}$.

An emerging area of interest is this relationship between the GI and respiratory tract, the 


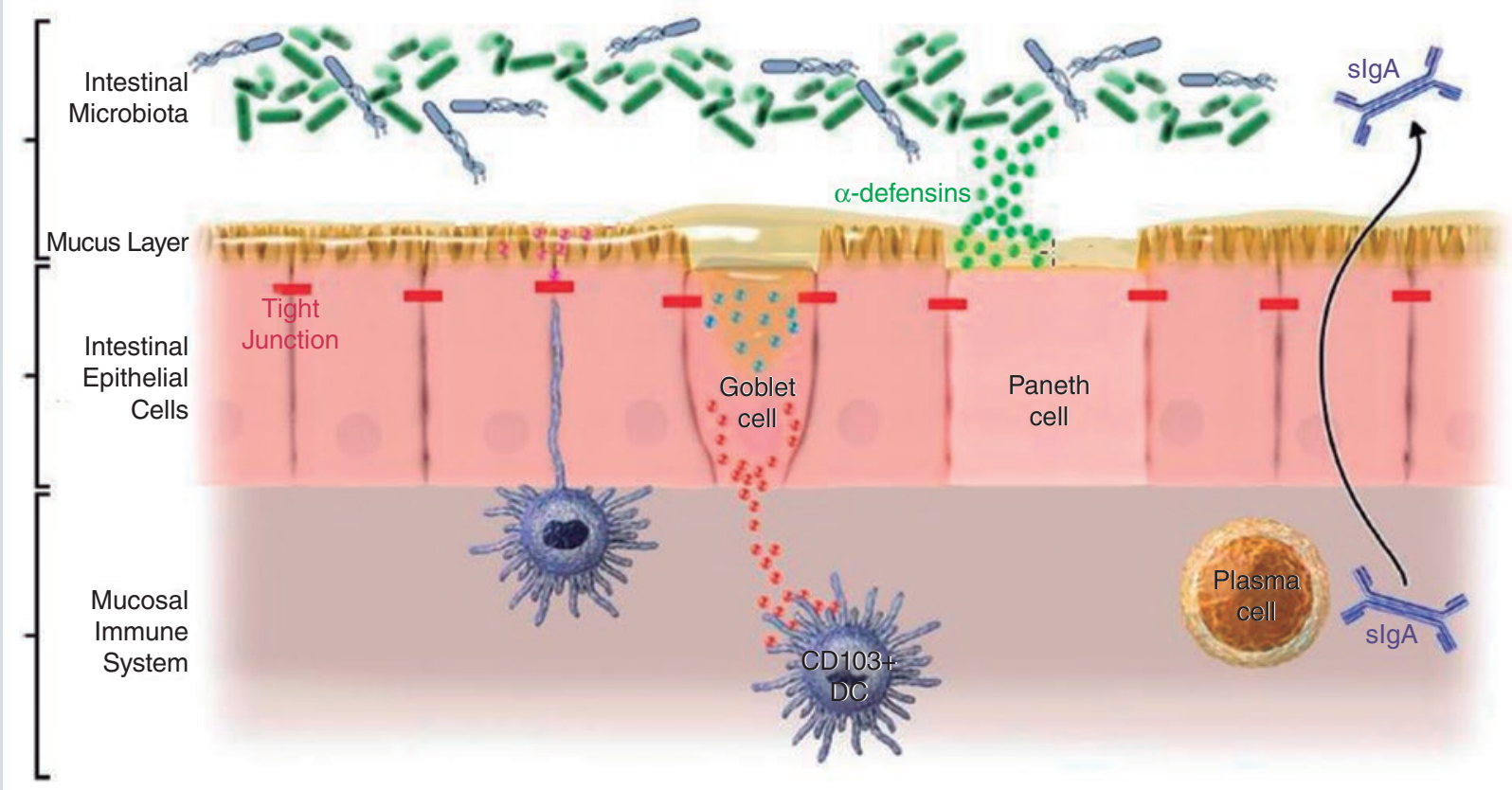

FIGURE 1. Structure of the intestinal barrier (adapted from Samadi $N$ et al..80, reproduced with permission from (c) 2018 American College of Allergy, Asthma \& Immunology).

slgA: soluble immunoglobulin A; DC: dendritic cells.

influence of host-associated microbiota on local and systemic immunity as well as the pathogenetic links between microbiota and this gutlung axis.

\section{MICROBIOTA OF THE HEALTHY GUT AND LUNGS}

Mammalian body surfaces exposed to the environment are covered by diverse microbiota. Their compositions depend on a multitude of different factors and include host genetics, environment and host immunity. Furthermore, the specific functional characteristics of the different body surfaces determine the number and the composition of their cognate microbiota ${ }^{12}$.
The characterisation of the microbiome is an important initial step in understanding the role of the microbiome to health and disease. Each healthy adult typically harbours more than 1000 species of bacteria belonging to a relatively few known bacterial phyla with Bacteroidetes and Firmicutes being the dominant lineages (phyla) of the domain Bacteria ${ }^{13}$.

The GI tract is the best studied host-associated microbial ecosystem. The human intestine harbours nearly 100 trillion micro-organisms composed of more than 1000 distinct bacterial species ${ }^{14}$ and is by far the most densely colonised surface of the human body. Weighing approximately $1.5 \mathrm{~kg}$, microbial residents in the GI tract outnumber human cells about 
10-fold and genome size 100-fold ${ }^{15}$. Variations along the GI tract are governed by the prevailing environment, including $\mathrm{pH}$, the concentration of bile acids, retention time of digesta, mucin properties and host defence factors ${ }^{16}$. Therefore, there is considerable variation in the constituents of the gut microbiota among apparently healthy individuals ${ }^{17}$. Four bacterial phyla dominate the GI tract: the most abundant phylum is Bacteroidetes, followed by Firmicutes. Proteobacteria and Actinobacteria are the other two dominant phyla ${ }^{18,19}$. Different factors modulate the variation of the gut microbiome in humans as illustrated in figure 2. While gut microbiota compositions on species level differ between individuals, a core microbiome exists, which fulfils essential metabolic functions ${ }^{20}$. Briefly, fermentation of certain non-digestible dietary compounds and endogenous mucus, absorption of ions, as well as production of vitamins belong to the key functions of intestinal microbiota ${ }^{21}$. Bacterial numbers increase successively between upper and lower intestinal tract and fermentation processes are most intense in cecum and proximal colon ${ }^{12}$.

The lungs have a large surface area of $>130 \mathrm{~m}^{2}$ consisting of around 100,000 small airways and 200 million alveoli. The large surface responsible for oxygen uptake is concomitantly exposed to numerous micro-organisms such as viruses, bacteria or fungi, particles, allergens, pollutants and tobacco smoke. The lungs are therefore equipped with effective antimicrobial defences. Healthy lungs were long considered to be sterile but culture-independent approaches have detected microbial DNA in the lungs of healthy individuals ${ }^{22,23}$. With an estimated number of 10-100 bacteria per 1000 human cells, the lower respiratory tract is one of the least-populated surfaces of the human body ${ }^{24}$. The composition of the lung microbiome is determined by the balance of three factors: (1) microbial immigration into the airways; (2) elimination of microbes from the airways; and (3) the relative reproduction rates of its community members, as determined by regional growth conditions. Sources of microbial immigration include the inhalation of air, subclinical microaspiration of upper respiratory contents and direct dispersal along the airway mucosa. Microbial elimination is driven by mucociliary clearance, cough and adaptive and innate immune defences ${ }^{25}$. Temperature, oxygen tension, $\mathrm{pH}$, nutrient density, local anatomy, and host defence are spatially heterogeneous across the airways and the lungs affecting local microbiological growth conditions $^{15}$ (Fig. 3).

Similar to the intestine, Firmicutes and Bacteroidetes are the two predominant phyla detected in the airways and core microbiota of healthy individuals consists of Pseudomonas, Streptococcus, Prevotella, Fusobacteria, Veillonella, Haemophilus, Neisseria and Porphyromonas ${ }^{26-29}$. The lung microbiota is probably not resident in healthy individuals but rather transiently recolonised by micro-aspiration from the oral cavity: the ubiquity of subclinical micro-aspiration of pharyngeal secretions among healthy subjects is a long-established and validated observation $^{30}$.

\section{THE GUT-LUNG AXIS}

Gut microbiota are considered crucial for the proper development, maturation and reactivity of the immune system. Secreted and structural components of micro-organisms can influence 
Figure 2. Determining factors of the gut microbiome.

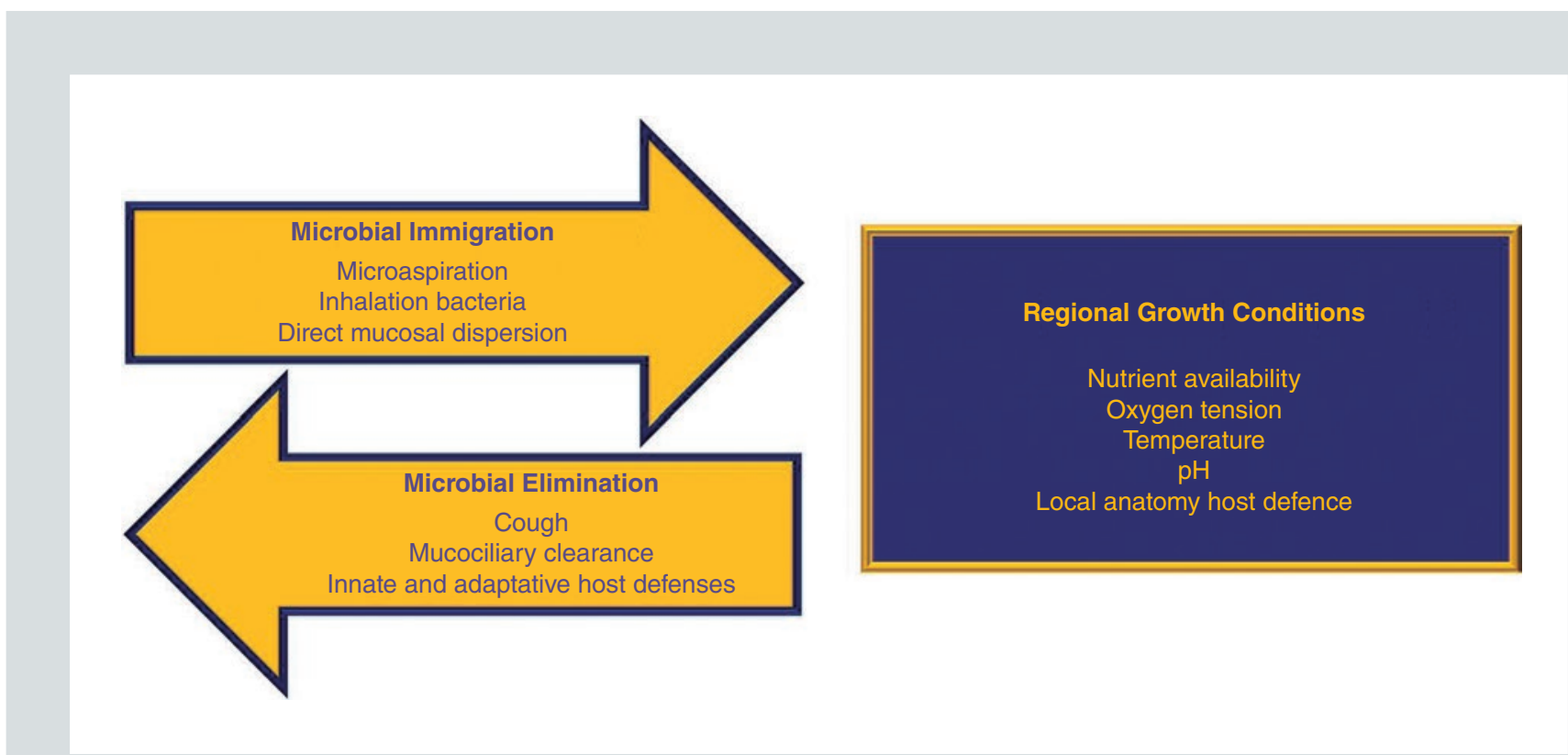

Figure 3. Determining factors of the lung microbiome (adapted from Dickson RP et al. ${ }^{25}$, reproduced with permission from (C) 2015 Dickson, Huffnagle).
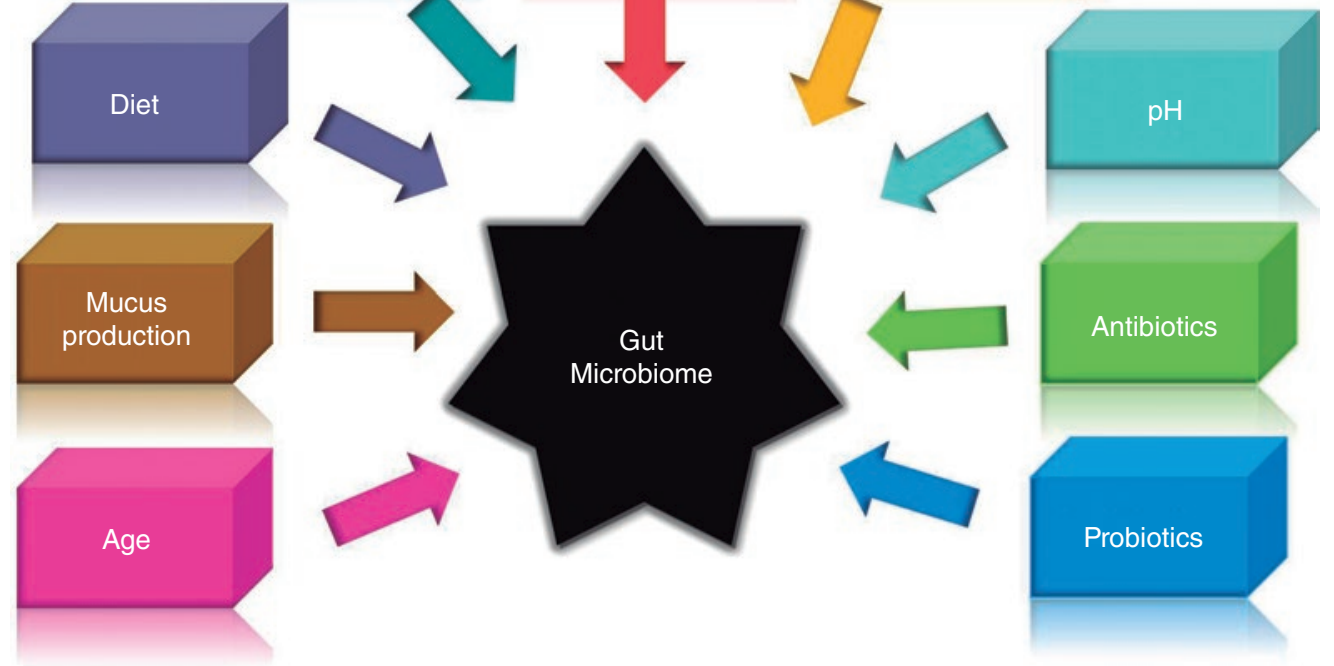

Gut

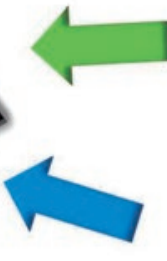

Antibiotics

Microbiome
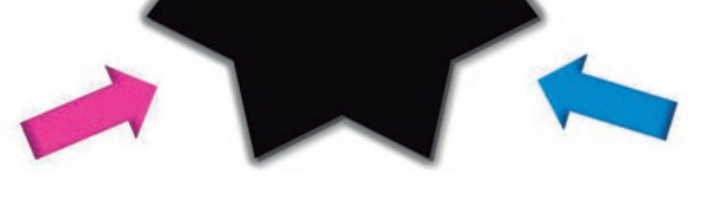

Probiotics 
the host immune response both locally and at distal sites. Micro-organisms serve as an inexhaustible source of micro-organism-associated molecular patterns (MAMPs) as well as pathogen-associated molecular patterns (PAMPs). The two are recognisable on the host's cells through pattern-recognition receptors (PRRs), which include toll-like receptors (TLRs) and nucleotide-binding receptors (NODs) ${ }^{15,31}$. Toll-like receptors are found on intestinal epithelial cells and immune cells within the lamina propria. Their activation in intestinal epithelial cells by microbial ligands results in epithelial cell proliferation and expression of antimicrobial peptides and secretion of immunoglobulin A (IgA) by plasma cells in the lamina propria leading to enhanced intestinal barrier function. Commensal micro-organisms can enter the intestinal lamina propria through an opening in the barrier function as a result of injury or through active sampling. These micro-organisms in the lamina propria are either phagocytosed and eliminated by macrophages or engulfed by DCs and carried to the mesenteric lymph nodes. Recognition of infected apoptotic cells and bacteria results in the differentiation and upregulation of pro-inflammatory helper-interleukin [IL]-17 producing cells (Th17), involved in the recruitment, activation and migration of neutrophils. Induction of B cells occurs also in the GI lymphoid tissue, particularly in Peyer's patches and in isolated lymphoid follicles. Specific populations of commensal bacteria can induce regulatory $\mathrm{T}$ cells $\left(\mathrm{T}_{\text {regs }}\right)$ to maintain homeostasis by production of anti-inflammatory cytokine IL-10 (Fig. 4).

These $\mathrm{T}$ and $\mathrm{B}$ cells can migrate via the thoracic duct and into the circulation. In this way, these sensitised $\mathrm{T}$ and $\mathrm{B}$ cells are distributed to various effector sites, including the respiratory tract to prevent attachment of pathogens to the mucosa by excretion of specific IgA resulting in a common mucosal immune defence system. The gut-associated lymphoid tissue (GALT) plays an important role in this mucosal immune system ${ }^{32}$. Nasopharynx-associated lymphoid tissue (NALT) exposed to both airborne and alimentary antigens has also a major role in antibody immunity of the respiratory tract.

Bacterial products that have a significant effect on overall host status surely included the short-chain fatty acids (SCFAs). These SCFAs are produced by metabolisation of resistant starches and dietary fibres through fermentation and decomposition. Fatty acids with a carbon number between 2 and 6 are considered SCFAs and have the following names: C2: acetic; C3: proprionic; C4: butyric; C5: valeric and C6: caproic acid ${ }^{33}$. These SCFAs account for $2-10 \%$ of the total energy consumption in humans and are the main energy source for large intestinal epithelial cells ${ }^{33}$. The concentration of SCFAs ranges between $70-140 \mathrm{mmol} / \mathrm{L}$ in the proximal colon and between $20-70 \mathrm{mmol} / \mathrm{L}$ in the distal colon ${ }^{34}$. Acetate is thought to be more prevalent, followed by proprionate and butyrate. Nearly all SCFAs absorbed by the colon are thought to pass through the portal vein from the colon capillaries to reach the li ver ${ }^{33}$. The concentrations of SFCAs in healthy human peripheral blood are estimated as 100-150 $\mu \mathrm{mol} / \mathrm{L}$ for acetate, 4-5 $\mu \mathrm{mol} / \mathrm{L}$ for proprionate and 1-3 $\mu \mathrm{mol} / \mathrm{L}$ for butyrate ${ }^{35}$.

The interplay between SFCAs and immune function is extensively studied $1,15,33,36,37$. Several G-protein coupled receptors (Gpr) for SCFAs have been reported: Gpr41/free fatty 

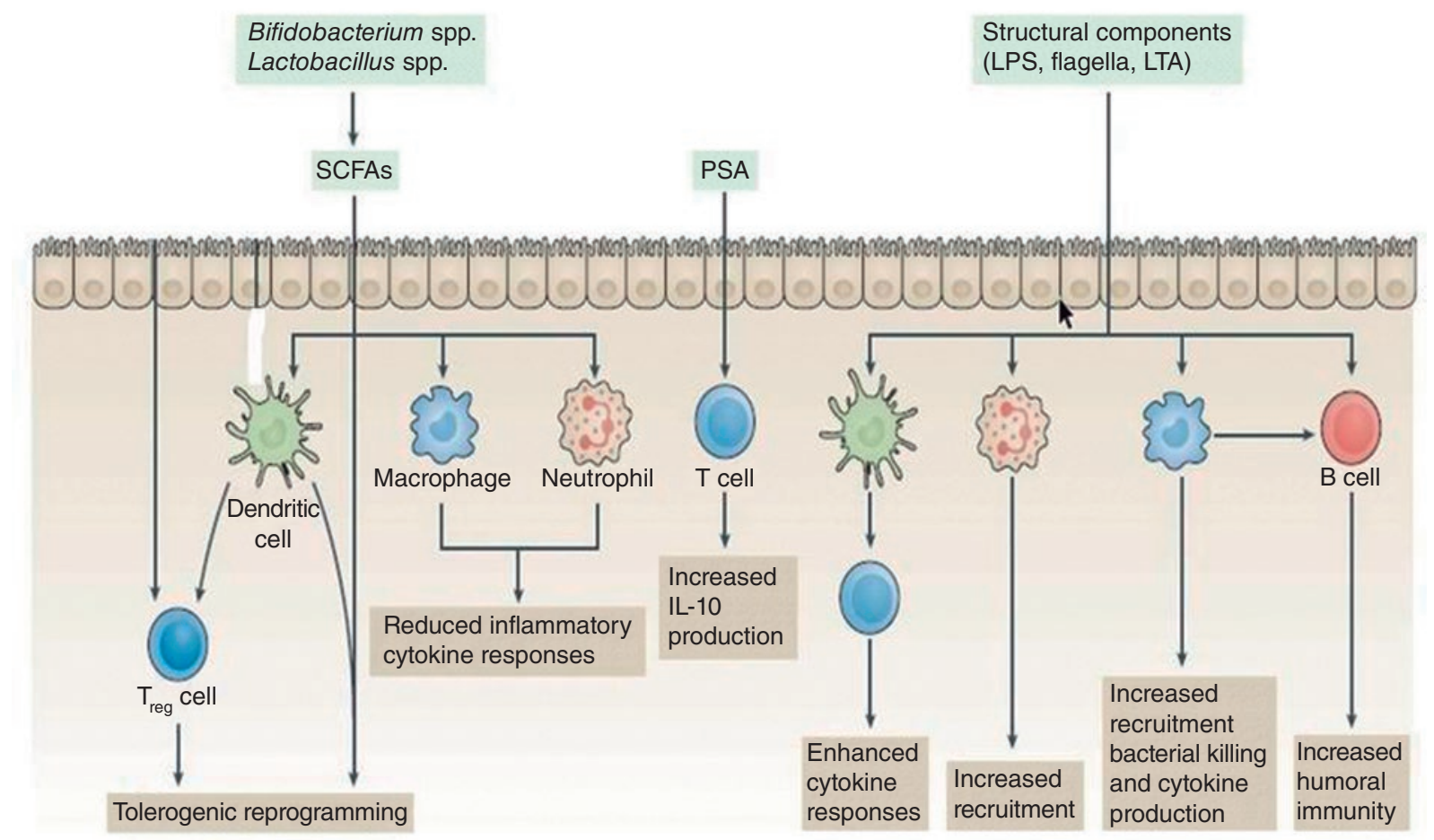

Figure 4. Immune system interaction and microbiota in the gut (adapted from Budden KF et al. ${ }^{1}$, reproduced with permission from Springer Nature, (C) 2016 Macmillan Publishers Limited, part of Springer Nature).

IL: interleukin; LPS: lipopolysaccharide; LTA: lipoteichoic acid; PSA: prostate-specific antigen; SCFAs: short-chain fatty acids; $\mathrm{T}_{\text {reg }}$ : regulatory $\mathrm{T}$ cell.

receptor (FFAR) 3, Gpr43/FFAR2, Gpr109A/ hydroxycarboxylic acid receptor (HCA) 2 and olfactory receptor (OR/Olfr) Olfr78 (mouse)/ OR51E2 (humans) (Fig. 5). Gpr41/FFAR3 is found in a wide range of tissues including neutrophils while Gpr43/FFAR2 is highly expressed on immune cells ${ }^{38}$. Gpr109A/HCA2 is a receptor to butyrate as well as $\beta$-hydroxybutyric acid, a ketone body and participates in regulation of $\mathrm{T}_{\text {reg }}$ homeostasis ${ }^{33}$. Butyrate manifests broad anti-inflammatory activities such as immune cell activation, proliferation, migration, adhesion and cytokine expression ${ }^{39}$. Short-chain fatty acids play an important role in $\mathrm{T}$ cell polarisation and induction ${ }^{40}$.

Short-chain fatty acids are widely known as histone deacetylase (HDAC) inhibitors and they may be involved in the expression of cytokines in $\mathrm{T}$ cells and the induction of $\mathrm{T}_{\text {reg }}$ cells via inhibition of $\mathrm{HDAC}^{41}$. Short-chain fatty acids also affect immunoregulation in neutrophils, monocytes and macrophages and suppression of nuclear factor-kappa B activity and inhibition of HDAC are possible underlying mechanisms but SFCAs have also promoting functions in neutrophils ${ }^{33}$. 


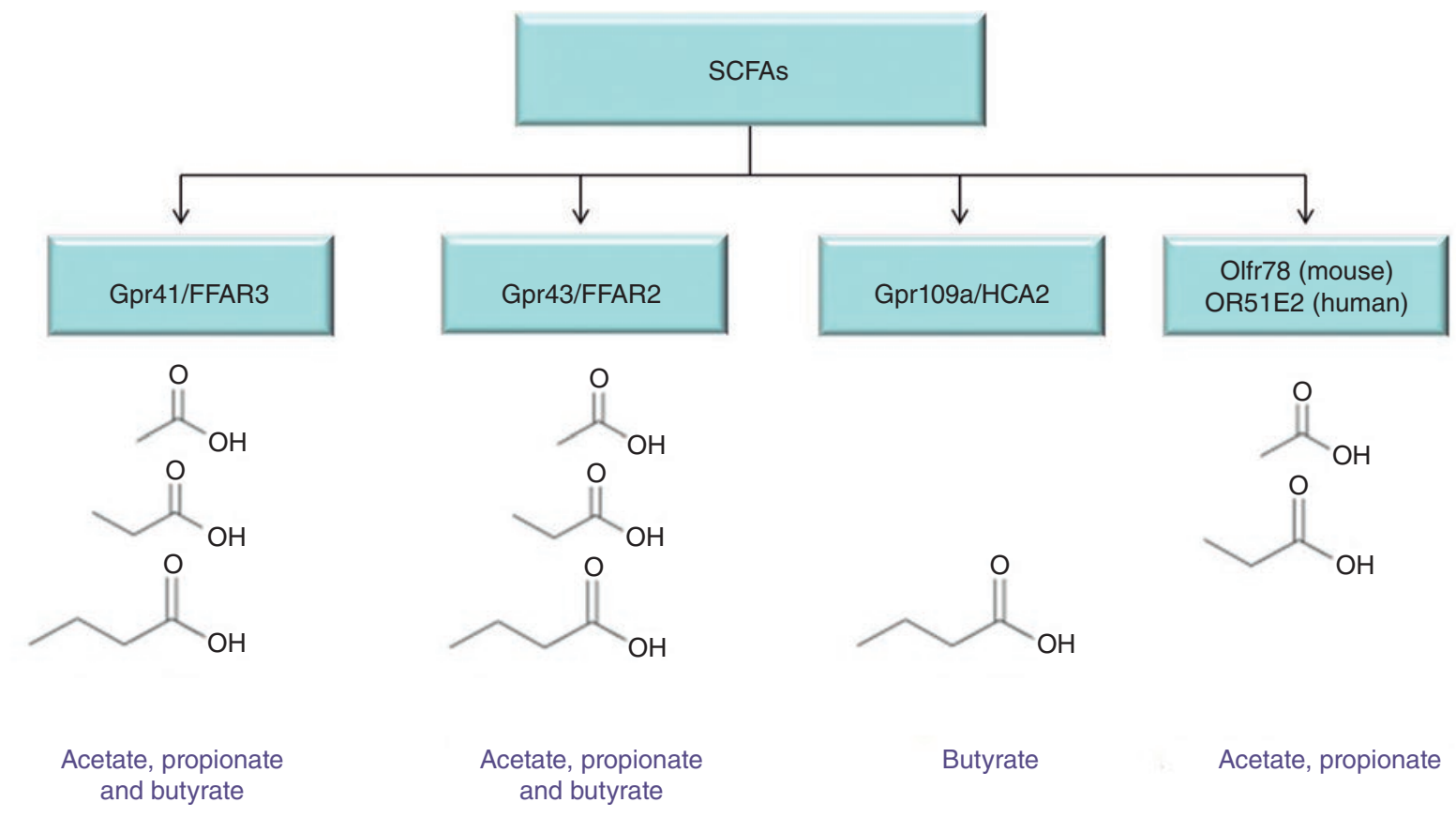

FIgURE 5. Short chain fatty acids and receptors.

FFAR: free fatty receptor; Gpr: G-protein coupled receptors; HCA: hydroxycarboxylic acid receptor; Olfr: olfactory receptor; 0R: olfactory receptor; SCFAs: short-chain fatty acids.

Intriguingly is the possible central role of SCFAs in the diet-gut microbiome-host metabolism axis and the protection of the body against deteriorating metabolic control and inflammatory status associated with western lifestyles ${ }^{42}$. Although not properly assessed, it can be hypothesised that bacterial products of the lung microbiota can exert their effects on the intestinal mucosa.

\section{MICROBIAL GUT DYSBIOSIS IN CHRONIC AIRWAY DISEASES}

Shifts in the composition of the intestinal microbiota have been reported in the context of chronic airway diseases as asthma, cystic fibrosis (CF) and COPD. The term dysbiosis refers to a state in which changes in the diversity and abundance of gut microbiota, their metabolic activities and local distribution produces harmful effects.

\section{a. Asthma}

Increased risk of asthma has been associated with reduced microbial diversity in the gut during early infancy ${ }^{37,43}$. Other allergic conditions as rhino-conjunctivitis, eczema or positive skin test reactivity were not associated with this gut diversity neither was asthma 
associated with microbiota composition later in life $\mathrm{f}^{43}$. Others reported that reduced bacterial diversity of the infant's intestinal flora was associated with increased risk of allergic sensitisation, allergic rhinitis and peripheral blood eosinophilia but not asthma or atopic dermatitis in the first six years of life ${ }^{44}$. In infants, the composition of the gut microbiota and caesarean section have been linked to atopic manifestations and colonisation with Clostridium difficile at one month of age was associated with wheeze and eczema throughout early life and with asthma after 6-7 years ${ }^{45}$. Increased risk of asthma has also been associated with an increase in Bacteroides fragilis and total anaerobes in early life, decreases in Escheria Coli and relative abundances of Faecalibacterium spp, Lachnospira spp., Rothia spp. and Veillonella spp. ${ }^{46-48}$. In the latter study, this reduction in bacterial taxa was accompanied by reduced levels of faecal acetate and dysregulation of enterohepatic metabolites. Although these studies emphasise the potential for microbe-based diagnostics and therapies, potentially in the form of probiotics, to prevent the development of asthma and other related allergic diseases in children, only inoculation of germ-free mice with the bacterial taxa reported by Arrieta et al. ${ }^{48}$ ameliorated airway inflammation in these animals. Recurrent antibiotic treatment during early infancy also impacts on the diversity of the microbiota early in life and has been shown to correlate with the development of an asthmatic phenotype later in life ${ }^{49,50}$. Positive associations between the presence of beneficial bacteria, such as Bifidobacterium longum in the gut and a lower incidence of asthma have also been identified ${ }^{51}$.

Furthermore, in mice it was illustrated that intestinal exposure to the capsular glycoantigen polysaccharide A(PSA), from Bacteroides Fragilis is a potent immunomodulator molecule, able to inhibit asthma induction in a T-cell dependent fashion and that this protection is IL-10 dependent ${ }^{52}$. It has also been reported that Helicobacter pylori alleviates murine allergic airway disease through activation of $\mathrm{T}_{\text {reg }}$ cells or by promoting tolerogenic reprogramming of DCs ${ }^{53,54}$. In adults, the overall composition of the faecal microbiota in individuals with allergic asthma does not differ from healthy individuals ${ }^{55,56}$.

The development of asthma can be influenced by SCFAs. An association between asthma risk and decrease in concentration of acetate in faeces in infants has been reported ${ }^{48}$. In mice, a high-fibre diet resulted in increased circulating levels of SCFAs and protection against allergic inflammation in the lung, whereas a low-fibre diet decreased levels of SCFAs and increased allergic airway disease. Treatment of mice with SCFAs proprionate led to alterations in bone marrow haematopoiesis that were characterised by enhanced generation of macrophage and DC precursors and subsequent seeding of the lungs by DCs with high phagocytic capacity but an impaired ability to promote T helper type 2 (TH2) cell effector function and these effects were dependent on GPR41/FFAR3 but not GPR42/FFAR $2^{57}$.

Short-chain fatty acids receptors are considered as a highly "druggable" target. In the future, such receptor-targeted approaches may offer complementary therapies in the inflammatory management of asthma ${ }^{1}$. Longitudinal and intervention studies need to unravel whether these changes in gut and respiratory microbiota are a cause or consequence in asthma. 


\section{b. Cystic fibrosis}

Cystic fibrosis (CF) is the most common life-limiting autosomal recessive disease in Caucasians and causes multisystem complications with progressive lung disease, pancreatic insufficiency and a profound impact on growth and nutrition. Cystic fibrosis transmembrane conductance regulator gene dysfunction itself modifies the ecological environment in the gut by impaired neutralisation of gastric bile ${ }^{58}$. Serial analysis of the gut and respiratory microbiome in CF in infancy demonstrated that distinct genera dominated in the gut compared to those in the respiratory tract with a core microbiota dominated by Veillonella and Streptococcus. Bacterial diversity increased significantly over time, with evidence of more rapid acquired diversity in the respiratory tract. Interestingly, changes in diet also resulted in altered respiratory microflora, suggesting a link between nutrition and microbial communities in the respiratory tract. These data illustrate that even in infants with $\mathrm{CF}$, nutritional factors and gut colonisation patterns are determinants of the microbial development of the respiratory tract microbiota ${ }^{59}$.

Compared with healthy controls, children with CF have significantly different intestinal microbial core structures. The levels of Eubacterium rectale, Bacteroides uniformis, Bacteroides vulgatus, Bifidobacterium adolescentis, Bifidobacterium catenulatum and Faecalibacterium prausnitzii were reduced in children with CF. A similar but more extreme pattern was observed in children with CF who were taking antibiotics. Interestingly, administration of Lactobacillus GG partially restored intestinal microbiota ${ }^{60}$. Comparison of the predominant faecal microbiota compositions of a group of paediatric
CF patients and their healthy siblings demonstrated that general medium counts, as well as counts on media specific for lactic acid bacteria, Clostridia, Bifidobacterium spp, Veillonella spp. and Bacteroides-Prevotella spp. were consistently higher in sibling samples than in CF samples, whereas the reverse was found for enterobacterial counts ${ }^{61}$.

A recent study identified the role of the CF mutation on the CF microbiota and demonstrated that those who were homozygous-F508del had more altered faecal microbiota compared to the other CF genotypes ${ }^{62}$. Studies on gut microbiota in stable adults with CF demonstrate significant altered microbiota, including reduced microbial diversity, an increase in Firmicutes and reduction of Bacteroidetes compared to the non-CF control population. Furthermore, CF patients presenting with severe lung dysfunction had significantly reduced gut microbiota diversity relative to those presenting with mild or moderate dysfunction and a significant negative correlation was observed between the number of intravenous antibiotic courses and gut microbiota diversity ${ }^{63}$.

The influence of gut microbes on respiratory immunity is further illustrated by the effects of ingested probiotics and prebiotics on respiratory infections and diseases. Probiotics have been shown to reduce the incidence of CF pulmonary exacerbations ${ }^{64,65}$. Previous studies already indicate that probiotic administration in CF also reduces the intestinal inflammation in these patients ${ }^{66}$.

These data illustrate the need to determine opportunities to minimise the disruption to the CF gut microbiota that occurs due to the 
disease and its management to influence lung disease outcomes.

\section{c. Chronic obstructive pulmonary disease}

Changes in gut microbiota as well as the interplay between gut and lung microbiota is poorly studied in patients with COPD. One study analysed respiratory tract microbiome in healthy smoking and non-smoking subjects ${ }^{21}$. The authors reported that lung bacterial communities resemble those in the oral cavity but also that the mouth microbiome differed between smokers and non-smokers in species such as Prophyromonas, Neisseria and Gemella. Porphyromonas, linked to periodontal disease, is generally increased in smokers who show a decreased inflammatory response to this organism ${ }^{67}$. At least, the data of this study suggest that smoking disrupts the normal community structure in the mouth. The possible link between these changes in mouth community and onset of disease development needs further research.

Smoking also disrupts the gut microbiota. Smokers have increased levels of Bacteroides-Prevorella than non-smokers: similar patterns are reported in patients with Crohn's disease ${ }^{68}$. The opposite seems also be true: smoking cessation substantially alters the intestinal microbiota: key representatives from the phyla of Firmicutes and Actinobacteria increase while Bacteriodetes and Proteobacteria decrease after a controlled stop smoking program ${ }^{69}$. The authors suggest a potential mechanistic association between these microbial shifts and alterations in body weight after smoking cessation. The causes of these smoking-associated changes in the composition of the gut microbiota were recently reviewed and are probably a combination of environmental, host and microbial changes ${ }^{1}$.

Intriguing is the role of Helicobacter pylori. While this pathogen has long been linked with decreased incidence of asthma and allergy, s2 meta-analyses suggested that infection with $H$. pylori is positively associated with an increased incidence of COPD and other chronic bronchial diseases ${ }^{70}$, and that systemic immune responses triggered by $H$. pylori have different roles in the aetiology of different lung disorders ${ }^{1}$. Longitudinal and intervention studies will be needed to unravel whether these changes in gut and respiratory microbiota are a cause or consequence of COPD.

\section{d. Exacerbations of chronic obstructive pulmonary disease}

He et al ${ }^{36}$ recently analysed the key conceptual features of exacerbations of chronic gut and lung diseases. They are both associated with profound bacterial dysbiosis and dysregulation, inducing host inflammatory responses: both exacerbation conditions include a disruption of the homeostatic balance between the resident organ microbiota and host immune mechanisms. Neither of the conditions have hallmarks of an acute infection, in which the pathogenetic bacterial species overtake a tissue site and contribute to tissue injury. In cases where antibiotics are beneficial, it is believed to be via manipulation of the bacterial community composition or indirect immunomodulatory effects of the antibiotics. 
Publications on the role of oral non-typeable Haemophilus influenzae (NTHi) immunisation with non-typeable Haemophilus influenzae have challenged the role of gut immunity in the pathophysiology of exacerbations of $\mathrm{COPD}^{71}$. Oral immunisation with inactivated NTHi in subjects with moderate-to-severe COPD had significant reductions in both positive sputum cultures and frequency of acute episodes without changes in specific $\operatorname{IgA}$ in secretions ${ }^{71}$. These observations contribute to the hypothesis that acute exacerbations occur as an uncontrolled and inappropriate inflammatory response to bacteria colonising damaged airways due to an ineffective Peyer's patch-derived T lymphocyte response and emphasise the critical role of the common mucosal system ${ }^{72}$ (Fig. 6). It is of note that aspiration of the bronchus content may deliver up to $10^{11}$ bacteria per day in the gut and a prospective study in smokers over a winter period showed a significant seasonal increase in antigen-reactive T-cells, consistent with the idea that swallowed sputum leads to antigen presentation to the GALT as a physiological mechanism of antigen handling ${ }^{73,74}$. Intriguing in this context is the imbalance of Th17 and $\mathrm{T}_{\text {reg }}$ cells and their respective cytokines in COPD patients in different stages, as observed in various autoimmune disorders. These cells are normally in a state of balance; if this balance is shifted toward Th17 cells, inflammatory processes are triggered throughout the body ${ }^{75}$. It has been demonstrated that the proportion of Th17 cells in peripheral blood and secreted Il-17 and TGF- $\beta 1$ levels in sputum are significantly higher in acute exacerbations of COPD than in stable COPD patients and controls, indicating that acute exacerbations of COPD are associated with a shifting toward a pro-inflammatory response with imbalance between Th17/ $\mathrm{T}_{\text {reg }}$ cells $^{76}$. These Th17 CD4+ lymphocytes produce IL-17A that promotes the activation of bronchial fibroblasts, epithelial cells and smooth muscle cells, and induces them to produce pro-inflammatory cytokines responsible for the recruitment of neutrophils and their local infiltration, aggravating COPD symptomatology ${ }^{77}$.

Immunotherapy with inactivated NTHi is assumed to augment this physiological loop based on aspiration content into the gut by stimulation of T-cells in the Peyer's patches of the GI tract to improve airway immune function $^{72}$. Although this concept is very challenging, a systematic review concludes that the $H$. influenzae vaccine taken orally in patients with chronic bronchitis and COPD does not have a significant reduction in the number and severity of acute exacerbations ${ }^{78}$. It would be useful to assess further both the longitudinal outcomes after one and two years following vaccination using careful standardised measurement protocols in COPD patients as well as the assessment of effects of vaccination in high-risk populations not yet diagnosed with COPD as a means of establishing the prophylactic capabilities of such vaccination ${ }^{78}$.

\section{THE LEAKY GUT SYNDROME IN CHRONIC RESPIRATORY DISEASES}

The GI barrier function is crucial in maintaining homeostasis of the body and selective permeability of the intestinal barrier is essential for oral tolerance development. Appropriate immune response and overall health 

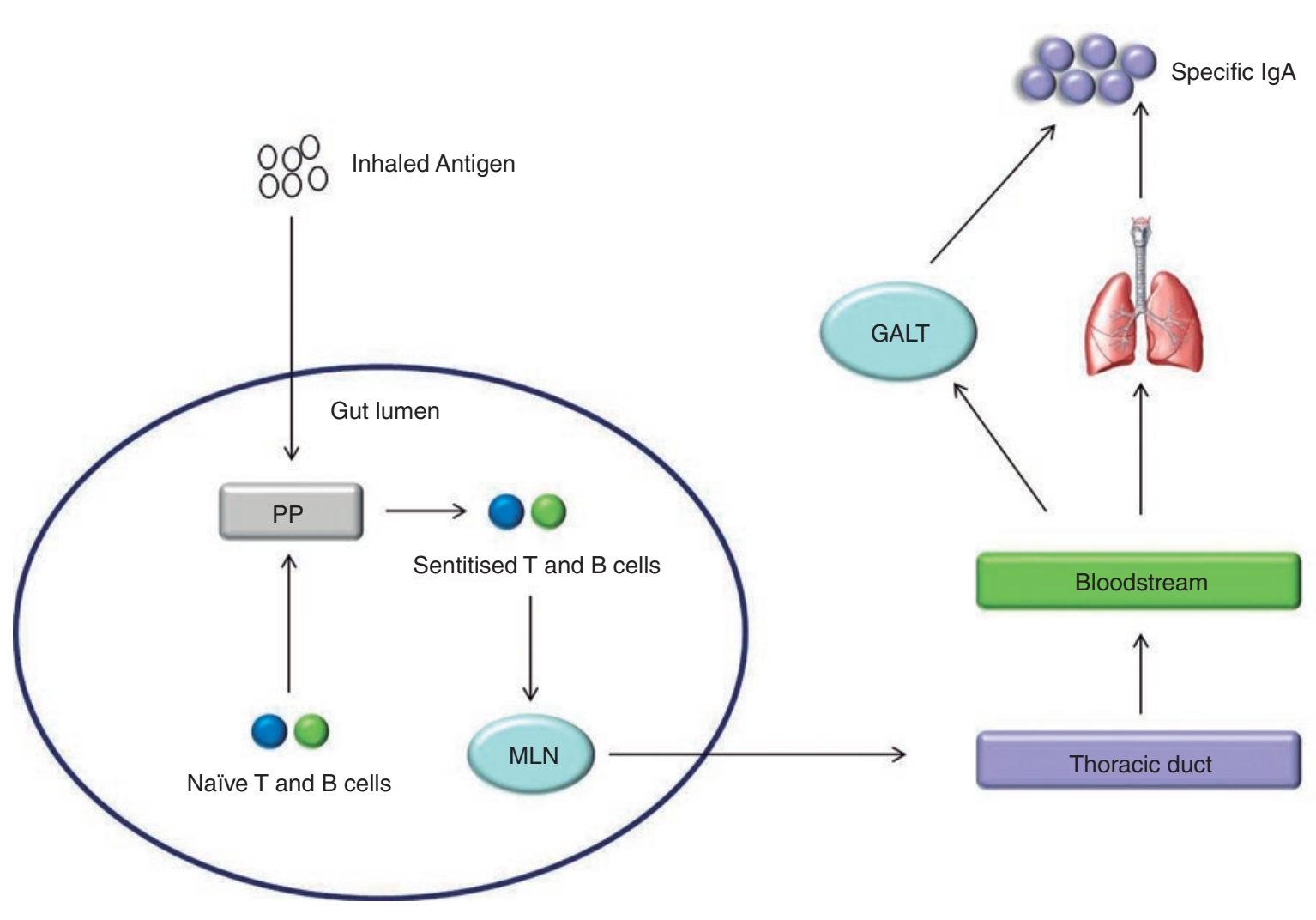

Figure 6. The common mucosal immune system (adapted from $\mathrm{He} \mathrm{Y}$ et al. ${ }^{36}$, reproduced with permission from Taylor \& Francis Ltd). GALT: gut-associated lymphoid tissue; Iga: immunoglobulin A; MLN: mesenteric lymph nodes; PP: Peyer's patches.

require a concerted interaction of the GI barrier, the host physiology and the GI luminal content. A number of intestinal and extra-intestinal diseases are described as being associated with a changed intestinal barrier function and increased permeability. The "leaky gut syndrome" is a phenomenon of increased intestinal permeability due to disruption of tight junctions.

On the basis of the concept that asthma is a T lymphocyte-mediated inflammatory disease involving the whole mucosal immune system, different studies reported an increased intestinal permeability in asthmatic children and adults ${ }^{79}$. A dysfunctional barrier of the entire GI tract has an essential contribution to food allergic reactions ${ }^{80}$.

Studies in CF patients showed evidence for a CF enteropathy, manifested by enterocyte damage and intestinal inflammation ${ }^{81,82}$. Furthermore, evidence is provided for an inverse correlation between this enteropathy and lung function impairment and priming of the small intestine to absorb microbial products potentially triggering hepatic inflammatory signalling and fibrogenesis pathways ${ }^{82}$. 
In COPD patients, it has been demonstrated that an altered intestinal permeability under resting conditions is intensified during daily life activities. These data suggest that COPD patients are unable to cope with the metabolic demand of daily life activities, probably related to intestinal ischaemia and associated enterocyte damage ${ }^{83}$. Intestinal compromise could be a contributing factor in systemic inflammatory changes in COPD. Increased small intestinal permeability has also been reported in COPD patients hospitalised for an acute exacerbation ${ }^{84}$.

Further studies will be needed to unravel these enteropathic changes in the different disease conditions but they also indicate that the gut can become an interesting future target for therapeutic interventions in chronic respiratory conditions. Intestinal epithelial cell turnover and gut barrier functions must be considered as dynamic processes, affected by nutritional status, route of feeding as well as the adequacy of specific nutrients in the diet.

\section{CONCLUSIONS AND PERSPECTIVES}

The gut and lung microbiota are essential to health and bacterial components and metabolites in the gut, and lungs have the capacity to modulate systemic and local immunity. Understanding the mechanisms that mediate the gut-lung cross-talk and the role of the gut and lung microbiota in mediating, maintaining and regulating this cross-talk will shed new perspectives in understanding chronic respiratory conditions and will potentially lead to identification of new and effective avenues for treatment. Future studies are needed to better discern causative effects and longitudinal studies must identify the role of these lung-gut interactions not only on the development of chronic respiratory conditions but also on their role in established chronic lung diseases. Future mechanistic microbiome studies should be directed towards understanding interactions between the host and microbes, using metagenomics, metabolomics and meta-transcriptomics to identify differentially expressed microbial genes in the patient's journey. Future studies will be needed to understand underlying mechanisms of the leaky gut syndrome in different chronic respiratory conditions. Gut dysfunction as well as gut-lung cross talks can become part of integrated management strategies in patients with chronic respiratory diseases.

\section{DISCLOSURES}

Dr. Wouters reports personal fees from $\mathrm{Ny}-$ comed, Boehringer, AstraZeneca, GSK, Novartis and Chiesi outside the submitted work.

\section{REFERENCES}

1. Budden KF, Gellatly SL, Wood DL et al. Emerging pathogenic links between microbiota and the gut-lung axis. Nat Rev Microbiol. 2017;15:55-63.

2. Roussos A, Koursarakos P, Patsopoulos D, Gerogianni I, Philippou N. Increased prevalence of irritable bowel syndrome in patients with bronchial asthma. Respir Med. 2003;97:75-9.

3. Baral V, Connett G. Acute intestinal obstruction as a presentation of cystic fibrosis in infancy. J Cyst Fibros. 2008;7:277-9.

4. Keely S, Hansbro PM. Lung-gut cross talk: a potential mechanism for intestinal dysfunction in patients with COPD. Chest. 2014;145:199-200.

5. Wang J, Li F, Wei H, Lian ZX, Sun R, Tian Z. Respiratory influenza virus infection induces intestinal immune injury via microbiota-mediated Th17 cell-dependent inflammation. J Exp Med. 2014;211:2397-2410.

6. Dilantika C, Sedyaningsih ER, Kasper MR et al. Influenza virus infection among pediatric patients reporting diarrhea and influenza-like illness. BMC Infect Dis. 2010;10:3.

7. Nielsen HM, Rodsgaard PA, Weinreich UM. Chronic obstructive pulmonary disease as comorbidity in patients admitted to a university hospital: a cross-sectional study. Clin Respir J. 2014;8:274-80. 
8. Rodriguez-Roisin R, Bartolome SD, Huchon G, Krowka MJ. Inflammatory bowel diseases, chronic liver diseases, and the lung (gut, liver and lung interplay). Eur Respir J 2016; 47: 638-50.

9. Ekbom A, Brandt L, Granath F, Lofdahl CG, Egesten A. Increased risk of both ulcerative colitis and Crohn's disease in a population suffering from COPD. Lung. 2008;186:167-72.

10. Yazar A, Atis S, Konca K et al. Respiratory symptoms and pulmonary functional changes in patients with irritable bowel syndrome. Am J Gastroenterol. 2001;96:1511-6.

11. Keely S, Talley NJ, Hansbro PM. Pulmonary-intestinal cross-talk in mucosal inflammatory disease. Mucosal Immunol. 2012;5:7-18.

12. Hauptmann M, Schaible UE. Linking microbiota and respiratory disease. FEBS Lett. 2016;590:3721-38.

13. Lozupone CA, Stombaugh JI, Gordon JI, Jansson JK, Knight R. Diversity, stability and resilience of the human gut microbiota. Nature. 2012;489:220-30.

14. Feng T, Elson CO. Adaptive immunity in the host-microbiota dialog. Mucosal Immunol. 2011;4:15-21.

15. Bingula R, Filaire M, Radosevic-Robin N et al. Desired Turbulence? GutLung Axis, Immunity, and Lung Cancer. J Oncol. 2017;2017:5035371.

16. Buffie CG, Pamer EG. Microbiota-mediated colonization resistance against intestinal pathogens. Nat Rev Immunol. 2013;13:790-801.

17. Human Microbiome Project C. Structure, function and diversity of the healthy human microbiome. Nature. 2012;486:207-14.

18. Eckburg PB, Bik EM, Bernstein $\mathrm{CN}$ et al. Diversity of the human intestinal microbial flora. Science. 2005;308:1635-8.

19. Robles-Alonso V, Guarner F. [Progress in the knowledge of the intestinal human microbiota]. Nutr Hosp. 2013;28:553-7.

20. Tap J, Mondot S, Levenez F et al. Towards the human intestinal microbiota phylogenetic core. Environ Microbiol. 2009;11:2574-84.

21. Guarner F, Malagelada JR. Gut flora in health and disease. Lancet. 2003; 361:512-9.

22. Morris A, Beck JM, Schloss PD et al. Comparison of the respiratory microbiome in healthy nonsmokers and smokers. Am J Respir Crit Care Med. 2013;187:1067-75.

23. Bassis CM, Erb-Downward JR, Dickson RP et al. Analysis of the upper respiratory tract microbiotas as the source of the lung and gastric microbiotas in healthy individuals. MBio. 2015;6:e0037.

24. Sze MA, Dimitriu PA, Hayashi S et al. The lung tissue microbiome in chronic obstructive pulmonary disease. Am J Respir Crit Care Med. 2012; 185:1073-80.

25. Dickson RP, Huffnagle GB. The Lung Microbiome: New Principles for Respiratory Bacteriology in Health and Disease. PLoS Pathog. 2015;11: e1004923.

26. Erb-Downward JR, Thompson DL, Han MK et al. Analysis of the lung microbiome in the "healthy" smoker and in COPD. PLoS One. 2011;6:e16384.

27. Hilty M, Burke C, Pedro H et al. Disordered microbial communities in asthmatic airways. PLoS One. 2010;5:e8578.

28. Charlson ES, Bittinger K, Haas AR et al. Topographical continuity of bacterial populations in the healthy human respiratory tract. Am J Respir Crit Care Med. 2011;184:957-63.

29. Charlson ES, Diamond JM, Bittinger K et al. Lung-enriched organisms and aberrant bacterial and fungal respiratory microbiota after lung transplant. Am J Respir Crit Care Med. 2012;186:536-45.

30. Gleeson K, Eggli DF, Maxwell SL. Quantitative aspiration during sleep in normal subjects. Chest. 1997;111:1266-72.

31. Ivanov, II, Honda K. Intestinal commensal microbes as immune modulators. Cell Host Microbe. 2012;12:496-508.

32. Brandtzaeg P. Secretory IgA: Designed for Anti-Microbial Defense. Front Immunol. 2013;4:222.

33. Ohira H, Tsutsui W, Fujioka Y. Are Short Chain Fatty Acids in Gut Microbiota Defensive Players for Inflammation and Atherosclerosis? J Atheroscler Thromb. 2017;24:660-72.

34. Wong JM, de Souza R, Kendall CW, Emam A, Jenkins DJ. Colonic health: fermentation and short chain fatty acids. J Clin Gastroenterol. 2006;40:235-43.
35. Cummings JH, Pomare EW, Branch WJ, Naylor CP, Macfarlane GT. Short chain fatty acids in human large intestine, portal, hepatic and venous blood. Gut. 1987;28:1221-7.

36. He Y, Wen Q, Yao F, Xu D, Huang Y, Wang J. Gut-lung axis: The microbial contributions and clinical implications. Crit Rev Microbiol. 2017;43:81-95.

37. Marsland BJ, Trompette A, Gollwitzer ES. The Gut-Lung Axis in Respiratory Disease. Ann Am Thorac Soc. 2015;12 Suppl 2:S150-6.

38. Le Poul E, Loison C, Struyf S et al. Functional characterization of human receptors for short chain fatty acids and their role in polymorphonuclear cell activation. J Biol Chem. 2003;278:25481-9.

39. Singh N, Gurav A, Sivaprakasam S et al. Activation of Gpr109a, receptor for niacin and the commensal metabolite butyrate, suppresses colonic inflammation and carcinogenesis. Immunity. 2014;40:128-39.

40. Belkaid Y, Hand TW. Role of the microbiota in immunity and inflammation. Cell. 2014;157:121-41.

41. Arpaia N, Campbell C, Fan X et al. Metabolites produced by commensal bacteria promote peripheral regulatory T-cell generation. Nature. 2013;504:451-5.

42. Morrison DJ, Preston T. Formation of short chain fatty acids by the gut microbiota and their impact on human metabolism. Gut Microbes. 2016;7 189-200.

43. Abrahamsson TR, Jakobsson HE, Andersson AF, Bjorksten B, Engstrand L, Jenmalm MC. Low gut microbiota diversity in early infancy precedes asthma at school age. Clin Exp Allergy. 2014;44:842-50.

44. Bisgaard H, Li N, Bonnelykke K et al. Reduced diversity of the intestinal microbiota during infancy is associated with increased risk of allergic disease at school age. J Allergy Clin Immunol. 2011;128:646-52.e1-5.

45. van Nimwegen FA, Penders J, Stobberingh EE et al. Mode and place of delivery, gastrointestinal microbiota, and their influence on asthma and atopy. J Allergy Clin Immunol. 2011;128:948-955.e1-3.

46. Vael C, Nelen V, Verhulst SL, Goossens H, Desager KN. Early intestinal Bacteroides fragilis colonisation and development of asthma. BMC Pulm Med. 2008;8:19.

47. Orivuori L, Mustonen K, de Goffau MC et al. High level of fecal calprotectin at age 2 months as a marker of intestinal inflammation predicts atopic dermatitis and asthma by age 6. Clin Exp Allergy. 2015;45:928-39.

48. Arrieta MC, Stiemsma LT, Dimitriu PA et al. Early infancy microbial and metabolic alterations affect risk of childhood asthma. Sci Transl Med. 2015 7:307ra152.

49. Fanaro S, Chierici R, Guerrini P, Vigi V. Intestinal microflora in early infancy: composition and development. Acta Paediatr Suppl. 2003;91:48-55

50. Fouhy F, Guinane CM, Hussey S et al. High-throughput sequencing reveals the incomplete, short-term recovery of infant gut microbiota following parenteral antibiotic treatment with ampicillin and gentamicin. Antimicrob Agents Chemother. 2012;56:5811-20.

51. Akay HK, Bahar Tokman H, Hatipoglu $\mathrm{N}$ et al. The relationship between bifidobacteria and allergic asthma and/or allergic dermatitis: a prospective study of 0-3 years-old children in Turkey. Anaerobe. 2014;28:98-103.

52. Johnson JL, Jones MB, Cobb BA. Bacterial capsular polysaccharide prevents the onset of asthma through T-cell activation. Glycobiology. 2015;25:368-75.

53. Sehrawat A, Sinha S, Saxena A. Helicobacter pylori neutrophil-activating protein: a potential Treg modulator suppressing allergic asthma? Front Microbiol. 2015;6:493.

54. Koch KN, Hartung ML, Urban S et al. Helicobacter urease-induced activation of the TLR2/NLRP3/IL-18 axis protects against asthma. J Clin Invest. 2015;125:3297-3302.

55. Hevia A, Milani C, Lopez P et al. Allergic Patients with Long-Term Asthma Display Low Levels of Bifidobacterium adolescentis. PLoS One. 2016;11: e0147809.

56. Hua X, Goedert JJ, Pu A, Yu G, Shi J. Allergy associations with the adult fecal microbiota: Analysis of the American Gut Project. EBioMedicine. 2016 3:172-9.

57. Trompette A, Gollwitzer ES, Yadava K et al. Gut microbiota metabolism of dietary fiber influences allergic airway disease and hematopoiesis. Nat Med. 2014;20:159-66. 
58. De Lisle RC, Borowitz D. The cystic fibrosis intestine. Cold Spring Harb Perspect Med. 2013;3:a009753.

59. Madan JC, Koestler DC, Stanton BA et al. Serial analysis of the gut and respiratory microbiome in cystic fibrosis in infancy: interaction between intestinal and respiratory tracts and impact of nutritional exposures. MBio. 2012;3: e00251-12. .

60. Bruzzese E, Callegari ML, Raia V et al. Disrupted intestinal microbiota and intestinal inflammation in children with cystic fibrosis and its restoration with Lactobacillus GG: a randomised clinical trial. PLoS One. 2014;9: e87796.

61. Duytschaever G, Huys G, Bekaert M, Boulanger L, De Boeck K, Vandamme P. Cross-sectional and longitudinal comparisons of the predominant fecal microbiota compositions of a group of pediatric patients with cystic fibrosis and their healthy siblings. Appl Environ Microbiol. 2011;77:8015-24.

62. Schippa S, Iebba V, Santangelo F et al. Cystic fibrosis transmembrane conductance regulator (CFTR) allelic variants relate to shifts in faecal microbiota of cystic fibrosis patients. PLoS One. 2013;8:e61176.

63. Burke DG, Fouhy F, Harrison MJ et al. The altered gut microbiota in adults with cystic fibrosis. BMC Microbiol. 2017;17:58.

64. Bruzzese E, Raia V, Spagnuolo MI et al. Effect of Lactobacillus GG supplementation on pulmonary exacerbations in patients with cystic fibrosis: a pilot study. Clin Nutr. 2007;26:322-8.

65. Weiss B, Bujanover Y, Yahav Y, Vilozni D, Fireman E, Efrati O. Probiotic supplementation affects pulmonary exacerbations in patients with cystic fibrosis: a pilot study. Pediatr Pulmonol. 2010;45:536-40.

66. Bruzzese E, Raia V, Gaudiello G et al. Intestinal inflammation is a frequent feature of cystic fibrosis and is reduced by probiotic administration. Aliment Pharmacol Ther. 2004;20:813-9.

67. Charlson ES, Chen J, Custers-Allen R et al. Disordered microbial communities in the upper respiratory tract of cigarette smokers. PLoS One. 2010;5:e15216.

68. Benjamin JL, Hedin CR, Koutsoumpas A et al. Smokers with active Crohn's disease have a clinically relevant dysbiosis of the gastrointestinal microbiota. Inflamm Bowel Dis. 2012;18:1092-1100.

69. Biedermann L, Brulisauer K, Zeitz J et al. Smoking cessation alters intestinal microbiota: insights from quantitative investigations on human fecal samples using FISH. Inflamm Bowel Dis. 2014;20:1496-1501.

70. Wang F, Liu J, Zhang Y, Lei P. Association of Helicobacter pylori infection with chronic obstructive pulmonary disease and chronic bronchitis: a meta-analysis of 16 studies. Infect Dis (Lond). 2015;47:597-603.
71. Clancy R, Cripps A, Murree-Allen K, Yeung S, Engel M. Oral immunisation with killed Haemophilus influenzae for protection against acute bronchitis in chronic obstructive lung disease. Lancet. 1985;2:1395-7.

72. Clancy RL, Dunkley M. Acute exacerbations in COPD and their control with oral immunization with non-typeable haemophilus influenzae. Front Immunol. 2011;2:7.

73. Lehmann D, Coakley KJ, Coakley CA et al. Reduction in the incidence of acute bronchitis by an oral Haemophilus influenzae vaccine in patients with chronic bronchitis in the highlands of Papua New Guinea. Am Rev Respir Dis. 1991;144:324-30.

74. Clancy RL, Dunkley ML. Oral non-typable Haemophilus influenzae enhances physiological mechanism of airways protection. Clin Exp Immunol. 2010;161:127-33.

75. Fromer L, Cooper CB. A review of the GOLD guidelines for the diagnosis and treatment of patients with COPD. Int J Clin Pract. 2008;62:1219-36.

76. Li XN, Pan X, Qiu D. Imbalances of Th17 and Treg cells and their respective cytokines in COPD patients by disease stage. Int J Clin Exp Med. 2014;7: 5324-29.

77. Ponce-Gallegos MA, Ramirez-Venegas A, Falfan-Valencia R. Th17 profile in COPD exacerbations. Int J Chron Obstruct Pulmon Dis. 2017;12:1857-65.

78. Teo E, House $\mathrm{H}$, Lockhart $\mathrm{K}$ et al. Haemophilus influenzae oral vaccination for preventing acute exacerbations of chronic bronchitis and chronic obstructive pulmonary disease. Cochrane Database Syst Rev. 2014(9):CD010010.

79. Hijazi Z, Molla AM, Al-Habashi H, Muawad WM, Molla AM, Sharma PN Intestinal permeability is increased in bronchial asthma. Arch Dis Child. 2004;89:227-9.

80. Samadi N, Klems M, Untersmayr E. The role of gastrointestinal permeability in food allergy. Ann Allergy Asthma Immunol. 2018;121:168-73.

81. Adriaanse MP, van der Sande LJ, van den Neucker AM et al. Evidence for a Cystic Fibrosis Enteropathy. PLoS One. 2015;10:e0138062.

82. Flass T, Tong S, Frank DN et al. Intestinal lesions are associated with altered intestinal microbiome and are more frequent in children and young adults with cystic fibrosis and cirrhosis. PLoS One. 2015;10:e0116967.

83. Rutten EPA, Lenaerts K, Buurman WA, Wouters EFM. Disturbed intestinal integrity in patients with COPD: effects of activities of daily living. Chest. 2014;145:245-52.

84. Sprooten RTM, Lenaerts K, Braeken DCW et al. Increased Small Intestinal Permeability during Severe Acute Exacerbations of COPD. Respiration. 2018;95:334-42. 